SLAC-PUB-12238

Nov. 2006

\title{
The Polarized Electron Source for the International Collider (ILC) Project ${ }^{*}$
}

\author{
A. Brachmann, J.E. Clendenin, E.L. Garwin, K. Ioakeimidi, R.E. Kirby, \\ T. Maruyama, C.Y. Prescott, J. Sheppard, J. Turner, F. Zhou
}

Stanford Linear Accelerator Center, 2575 Sand Hill Rd., Menlo Park, CA 94025, USA

\begin{abstract}
The ILC project will be the next large high energy physics tool that will use polarized electrons (and positrons). For this machine spin physics will play an important role. The polarized electron source design is based on electron injectors built for the Stanford Linear Collider (polarized) and Tesla Test Facility (un-polarized). The ILC polarized electron source will provide a $5 \mathrm{GeV}$ spin polarized electron beam for injection into the ILC damping ring. Although most ILC machine parameters have been achieved by the SLC or TTF source, features of both must be integrated into one design. The bunch train structure presents unique challenges to the source laser drive system. A suitable laser system has not yet been demonstrated and is part of the ongoing R\&D program for ILC at SLAC. Furthermore, ILC injector R\&D incorporates photocathode development, increasing available polarization, and improving operational properties in gun vacuum systems. Another important area of research and development is advancing the design of $\mathrm{DC}$ and $\mathrm{RF}$ electron gun technology for polarized sources. This presentation presents the current status of the design and outlines aspects of the relevant R\&D program carried out within the ILC community.
\end{abstract}

Keywords: polarized electron source, International Linear Collider.

PACS: $29.25 . \mathrm{Bx}, 29.27 . \mathrm{Hj}, 52$.

\section{GENERAL DESCRIPTION OF THE ILC POLARIZED ELECTRON SOURCE}

The conceptual design of the ILC Polarized Electron Source is shown in Fig. 1. The injector consists of 2 DC guns incorporating photocathodes illuminated by two Ti:Sapphire drive laser beams. At one time only one gun and laser system will be in operation, the second system provides redundancy to achieve high source availability. The produced long $\mathrm{e}^{-}$micro bunches $(\sim 2 \mathrm{~ns})$ are compressed by two sub-harmonic bunchers operating at $108 \mathrm{MHz}$ and $433 \mathrm{MHz}$, respectively. A $\beta$ matched traveling wave L-band buncher/pre-accelerator reduces the bunch length further to $\sim 20$ picosseconds (FWHM) and accelerates the beam to $\sim 80 \mathrm{MeV}$. A chicane provides energy collimation before the bunch train is injected into the superconducting booster linac for acceleration to $5 \mathrm{GeV}$. A transfer line connects the electron source with the damping ring. The bending angle is chosen to provide spin precession by 90 degrees. Superconducting solenoids are used to rotate the spin vector by 90 degrees into a

* This work is supported by Department of Energy contract DE-AC02-76SF00515 
SLAC-PUB-12238

Nov. 2006

vertical orientation prior injection into the damping ring. The source beam parameters for the ILC are summarized in table 1.

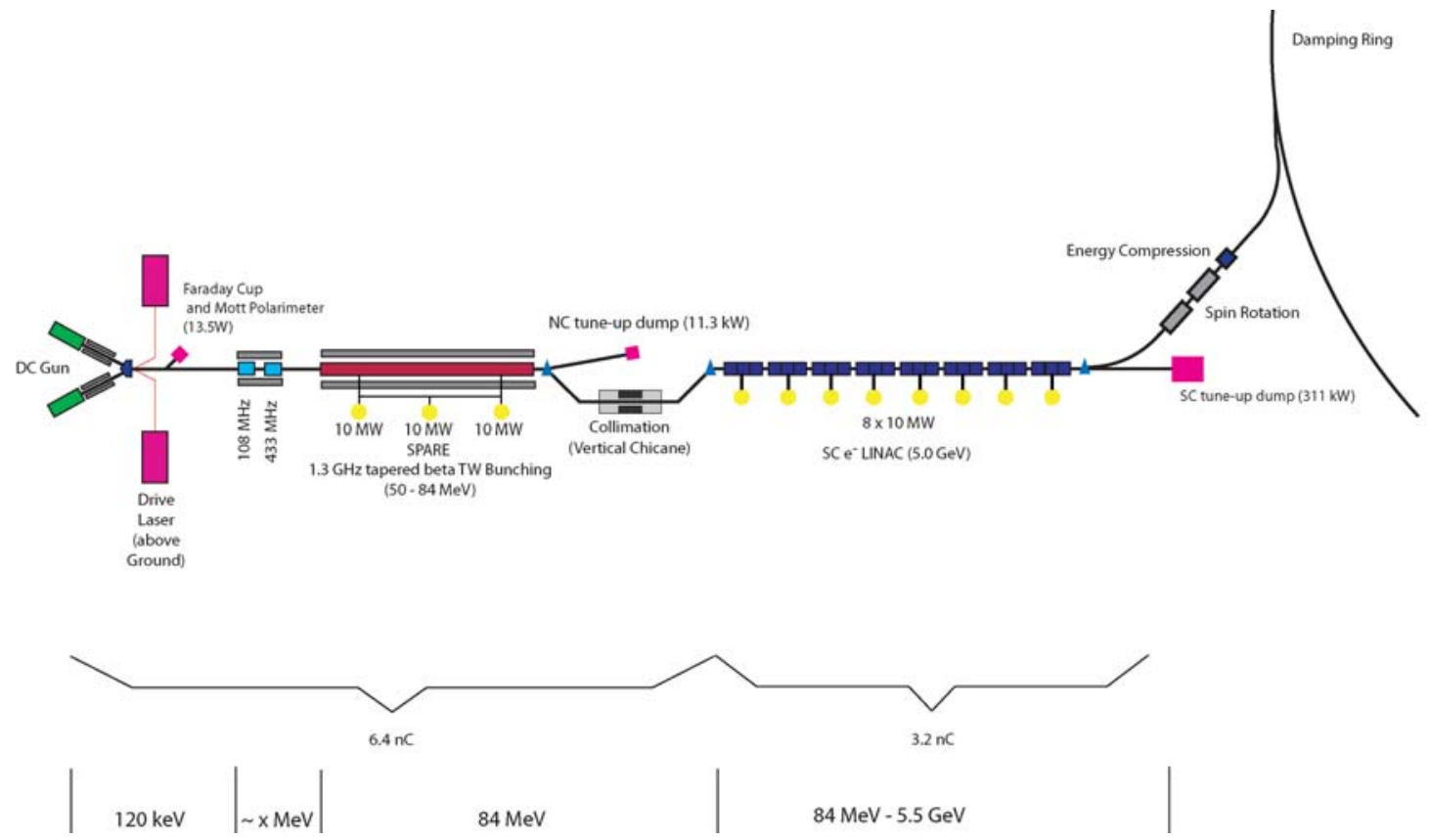

FIGURE 1. Schematic Layout of the ILC polarized electron source and injector systems.

\begin{tabular}{|l|l|l|l|}
\hline \multicolumn{1}{|c|}{ Parameter } & Symbol & Value & Unit \\
\hline Electrons per micro-bunch & $\mathrm{n}_{\mathrm{e}}$ & $4 * 10^{10}$ & Number \\
\hline Number of micro-bunches & $\mathrm{N}_{\mathrm{e}}$ & 2820 & Number \\
\hline Micro-bunch repetition rate & $\mathrm{F}_{\mu \mathrm{b}}$ & 3 & $\mathrm{MHz}$ \\
\hline Macro-bunch repetition rate & $\mathrm{F}_{\mathrm{mb}}$ & 5 & $\mathrm{~Hz}$ \\
\hline Micro-bunch charge at source & $\mathrm{C}$ & 6.4 & $\mathrm{nC}$ \\
\hline Micro-bunch length at source & $\Delta \mathrm{T}$ & 2 & $\mathrm{~ns}$ \\
\hline Peak current at source & $\mathrm{I}_{\mathrm{avg}}$ & 3.2 & $\mathrm{~A}$ \\
\hline Energy stability & $\mathrm{S}$ & $<5$ & $\% \mathrm{rms}$ \\
\hline Polarization & $\mathrm{P}_{\mathrm{e}}$ & 90 & $\%$ \\
\hline $\begin{array}{l}\text { Photocathode Quantum } \\
\text { Efficiency }\end{array}$ & $\mathrm{QE}$ & 1 & $\%$ \\
\hline Drive laser wavelength & $\lambda$ & $780( \pm 10)$ & $\mathrm{nm}$ \\
\hline Micro-pulse laser energy & $\mathrm{E}$ & 10 & $\mu \mathrm{J}$ \\
\hline
\end{tabular}

TABLE 1. Electron source system parameters requirement prior to injection in damping ring:

\section{ILC POLARIZED ELECTRON SOURCE R\&D}

The ILC Polarized Electron Source concept is based on the design of Stanford Linear Collider (SLC) source and incorporates the experience gained through 
subsequent polarized electron beam experiments at SLAC and elsewhere. However, the ILC presents unique challenges to the source such as the $1 \mathrm{~ms}$ long pulse train. This pulse train must be generated by the drive laser system. We expect the laser system to be based on Ti:Sapphire to provide wavelength tunability and sufficient bandwidth for temporal and spatial pulse shaping, which is desired to optimize source parameters such as emittance, space charge and beam loading effects. A laser system that can provide all requirements is currently beyond the state of the art and requires substantial R\&D (Fig. 2). Amplification of the pulse train will be the main challenge. Pre-amplification of a cavity dumped mode-locked pulse train will use diode pumped solid state laser technology. Additional flash-lamp pump amplifier stages will boost the pulse energy. The required laser pulse energy can be calculated using: $\mathrm{E}=\mathrm{ne} \cdot \mathrm{h} \cdot \mathrm{c} /(\mathrm{QE} \cdot \lambda)^{1}$. To compensate for $\mathrm{QE}$ decay between cesiations and for transport losses, the laser system must deliver $\sim 10 \mu \mathrm{J}$ per pulse.

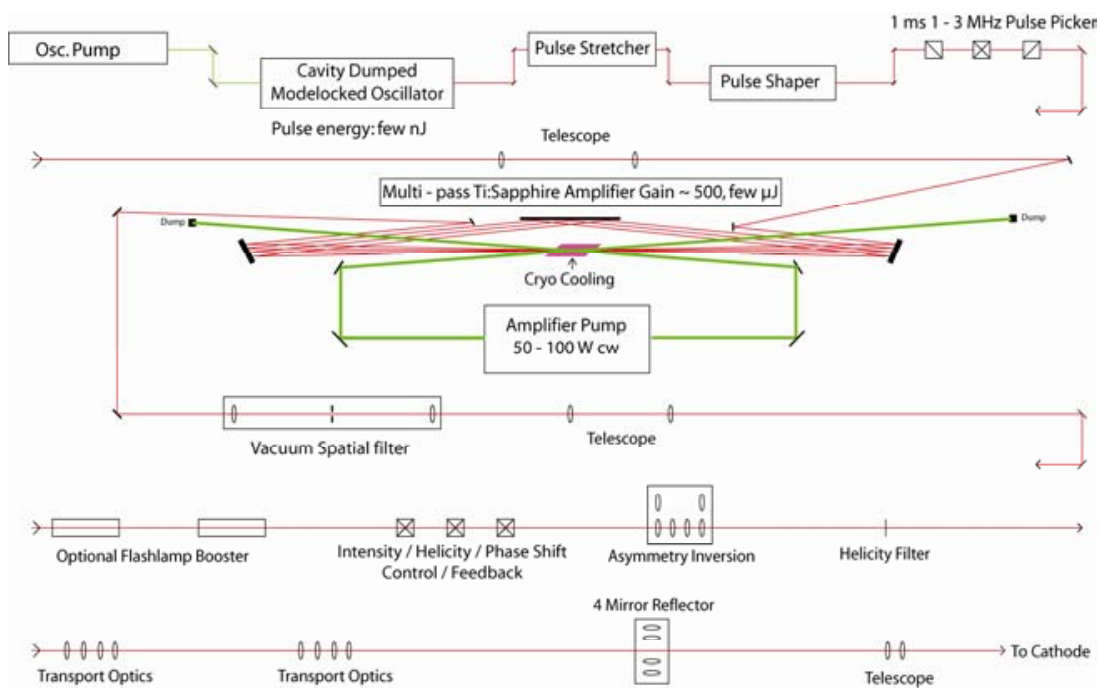

FIGURE 2. ILC Polarized Electron Source drive laser system schematic.

The ILC baseline design assumes a DC gun operating at $120 \mathrm{kV}$. Developments at other labs (e.g. ref. 1) have shown the feasibility of higher voltage guns. Details in regards to gun performance improvements resulting from increased bias voltage are summarized elsewhere in these proceedings (ref. 2). Clearly, a 'custom' designed higher voltage DC gun for the ILC injector will improve performance and overall operation of this machine. We anticipate significant research and design efforts will take place during the next years and results will be incorporated into the ILC technical design.

At SLAC, a strained-superlattice GaAs/GaAsP photocathode is installed in the main linac polarized gun. This cathode was initially installed in 2003 and provided beam for the last two months of the E158 experiment and subsequently has been used occasionally for the PEPII experiment. This cathode has been activated once when it was installed and cesiated in $\sim 1$ week intervals. Details regarding this cathode have

${ }^{1} \mathrm{E}$ : laser pulse energy, ne: number of electrons, c: speed of light, QE: quantum efficiency (fraction) and $\lambda$ : excitation wavelength 
SLAC-PUB-12238

Nov. 2006

been reported previously (ref. 3). Recently, the emission performance of the cathode has been evaluated. The results show that the cathode can still provide current in excess of ILC requirements (see Fig. 3). However, the QE profile indicates damage resulting from ion back-bombardment located near the center of the cathode (see Fig. 4). Similar results have been found at JLAB's polarized gun (ref.4). Consequently, the ILC sources R\&D program must include the design of robust cathodes, improved gun design (UHV and HV issues), and efforts must be undertaken to design an integrated load-lock system that facilitates efficient cathode replacement. Ongoing photocathode research is presented elsewhere in these proceedings( e.g. ref. 5,6).

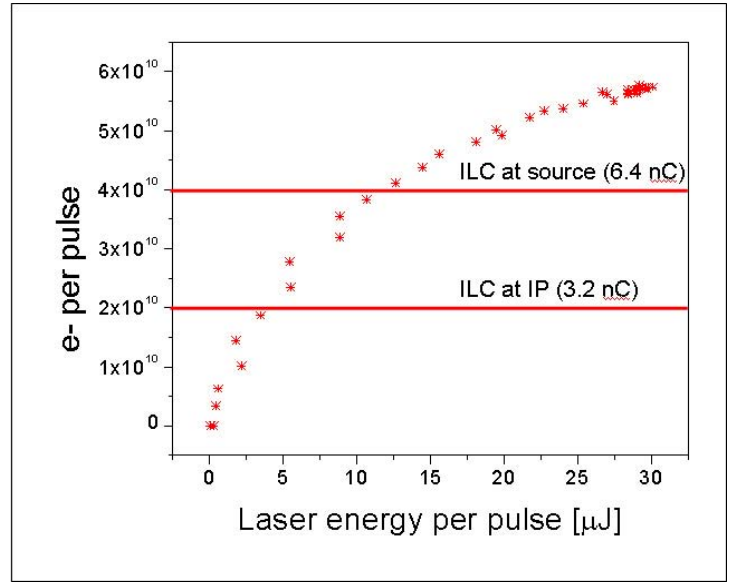

FIGURE 3. Extracted charge in a 2 ns pulse in August 2006 (after 3 years since installation of cathode)

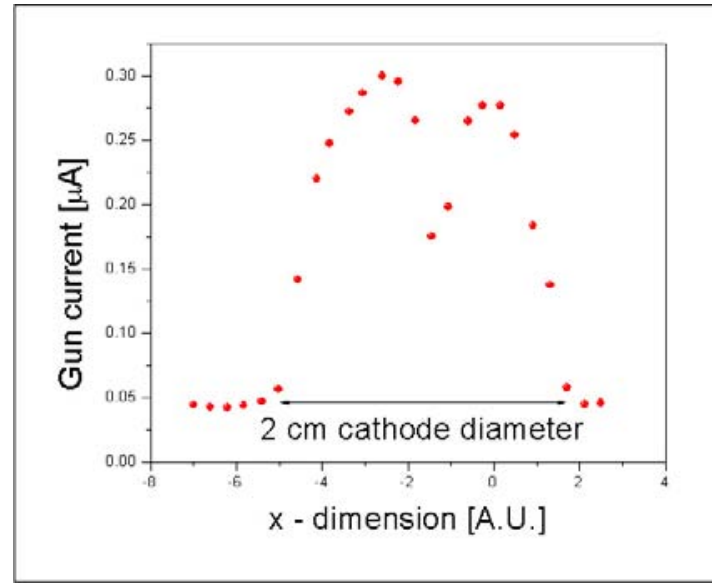

FIGURE 4. Cathode QE profile showing QE deterioration near cathode center (August 2006)

\section{SUMMARY}

The SLC injector provides a reasonable baseline for the ILC polarized electron source. The ILC sources R\&D program focuses on its further optimization. Key elements of the program are the laser system, DC gun with improved HV performance and ILC specific photocathode development. The main goal of the ILC source development is a 'proof of principle' demonstration of $\mathrm{e}^{-}$beam generation using an integrated laser/gun/photocathode system.

\section{References}

1 M. Yamamoto et al., High Field Gradient Polarized Electron Gun for ILC., PST05 proceedings.

2 J. Clendenin et al., Low Emittance Guns for the ILC Polarized Electron Beam. these proceedings.

3 A. Brachmann et al., PST05 Proceedings, SLAC-Pub-11711.

$4 \quad$ J. Grames et al., PAC05 Proceedings, p. 2875., 2005.

5 T. Maruyama et al., Polarization Possibilities of Small Spin-Orbit Interaction in StrainedSuperlattice Photocathodes, these proceedings.

6 K. Ioakeimidi et al., Transport Mechanisms in Polarized Semiconductor Photocathodes., these proceedings. 